\title{
Analysing Cell Level Allocation of Calcium and Phosphorus in Leaves of Proteaceae from South-Western Australia
}

Patrick E. Hayes ${ }^{1,2,3}$, Peta L. Clode ${ }^{2,1}$, Caio Guilherme Pereira ${ }^{1,2,4}$ and Hans Lambers ${ }^{1}$.

1. UWA School of Biological Sciences, The University of Western Australia. Perth, Australia.

2. Centre for Microscopy, Characterisation \& Analysis, The University of Western Australia. Perth, Australia.

3. Present Address: Crop, Livestock \& Environment Division, Japan International Research Center for Agricultural Sciences. Tsukuba, Japan.

4. Present Address: Department of Civil and Environmental Engineering, Massachusetts Institute of Technology. Cambridge, USA.

The plant family Proteaceae represent an iconic and ecologically important component of the Australian flora, contributing to south-western Australia's exceptionally high biodiversity and its status as a 'global biodiversity hotspot'. Most Proteaceae are highly phosphorus (P)-sensitive and occur exclusively on acidic soils with a very low $\mathrm{P}$ availability (calcifuge), whilst some also occur on young calcareous soils (soil-indifferent), higher in plant-available calcium (Ca) and P. The calcifuge habit of most Proteaceae can be explained by $\mathrm{Ca}$-enhanced $\mathrm{P}$ toxicity, which is putatively linked to the leaf cell-specific allocation of $\mathrm{Ca}$ and $\mathrm{P}$ [1]. Separation of these elements amongst cells is essential to avoid the deleterious precipitation of $\mathrm{Ca}$-phosphates, the formation of which reduces the availability of both nutrients and severely impacts cellular processes.

Species that have evolved in severely P-impoverished soils across south-western Australia, including Proteaceae, tend to allocate $\mathrm{P}$ to mesophyll cells and $\mathrm{Ca}$ to non-mesophyll cells, unlike many other eudicot species from P-richer habitats [2,3]. This represents an important adaptation, increasing P-use efficiency, but is also associated with P-toxicity. The low ability of these species to down-regulate P-uptake capacity, coupled with a preferential allocation of $\mathrm{P}$ to mesophyll cells, increases mesophyll $[\mathrm{P}]$, leading to $\mathrm{P}-$ toxicity. Calcium is thought to increase the severity of P-toxicity by further increasing the mesophyll [P]. However, this has never been quantitatively demonstrated. The aim of this research was to: 1) study the effects of $\mathrm{Ca}$ and $\mathrm{P}$ supply on the cell-specific allocation of these nutrients; and 2) compare responses between calcifuge and soil-indifferent species, linking this to differences in their distributions and sensitivity to Ca-enhanced $\mathrm{P}$ toxicity.

We used quantitative X-ray elemental microanalysis to determine leaf cell-specific nutrient concentrations in two calcifuge and two soil-indifferent Proteaceae species grown at a range of $\mathrm{Ca}$ and $\mathrm{P}$ concentrations in nutrient solutions. Quantitative X-ray mapping and elemental analyses were performed on frozenhydrated, transverse leaf sections using a Zeiss Supra 55 FESEM, fitted with a Leica VCT100 cryo-system and an Oxford Instruments X-Max80 SDD X-ray detector.

Our results show that $\mathrm{Ca}$ enhances the allocation of leaf $\mathrm{P}$ to palisade mesophyll cells, generally resulting in a greater mesophyll $[\mathrm{P}]$, providing the first quantitative demonstration of this interaction, and supporting the proposed mechanism of Ca-enhanced $\mathrm{P}$ toxicity. Calcifuges showed a greater response than soilindifferent species, with a greater palisade mesophyll $[\mathrm{P}]$, demonstrating the first mechanism able to explain their distribution. Through our comparison of calcifuge and soil-indifferent species we were also 
able to propose several strategies with which soil-indifferent species are able to tolerate high $\mathrm{Ca}$ and $\mathrm{P}$ supplies.

This study will assist in the management of this iconic plant family and other P-sensitive species, as it advances our understanding of the factors impacting their distribution, particularly the importance of $\mathrm{P}$ and $\mathrm{Ca}$. This is of increasing importance, as mining, agriculture, land-clearing, climate change, and other anthropogenic factors continue to influence Proteaceae distribution. This research contributes to our fundamental understanding of plant mineral nutrition and highlights the importance of considering interactions among essential macronutrients at the leaf tissue level and the benefits in utilizing new technologies to assess nutrient changes at the cellular level.

[1] PE Hayes et al, New Phytologist 221 (2019) p. 764-777.

[2] PE Hayes et al, Plant Cell and Environment 41 (2018) p. 605-619.

[3] C Guilherme Pereira et al, New Phytologist 218 (2018) p. 959-973.

[4] The authors acknowledge ARC Discovery Program funding (DP130100005) and the use of the equipment at CMCA, a facility funded by Universities, and State and Commonwealth governments.
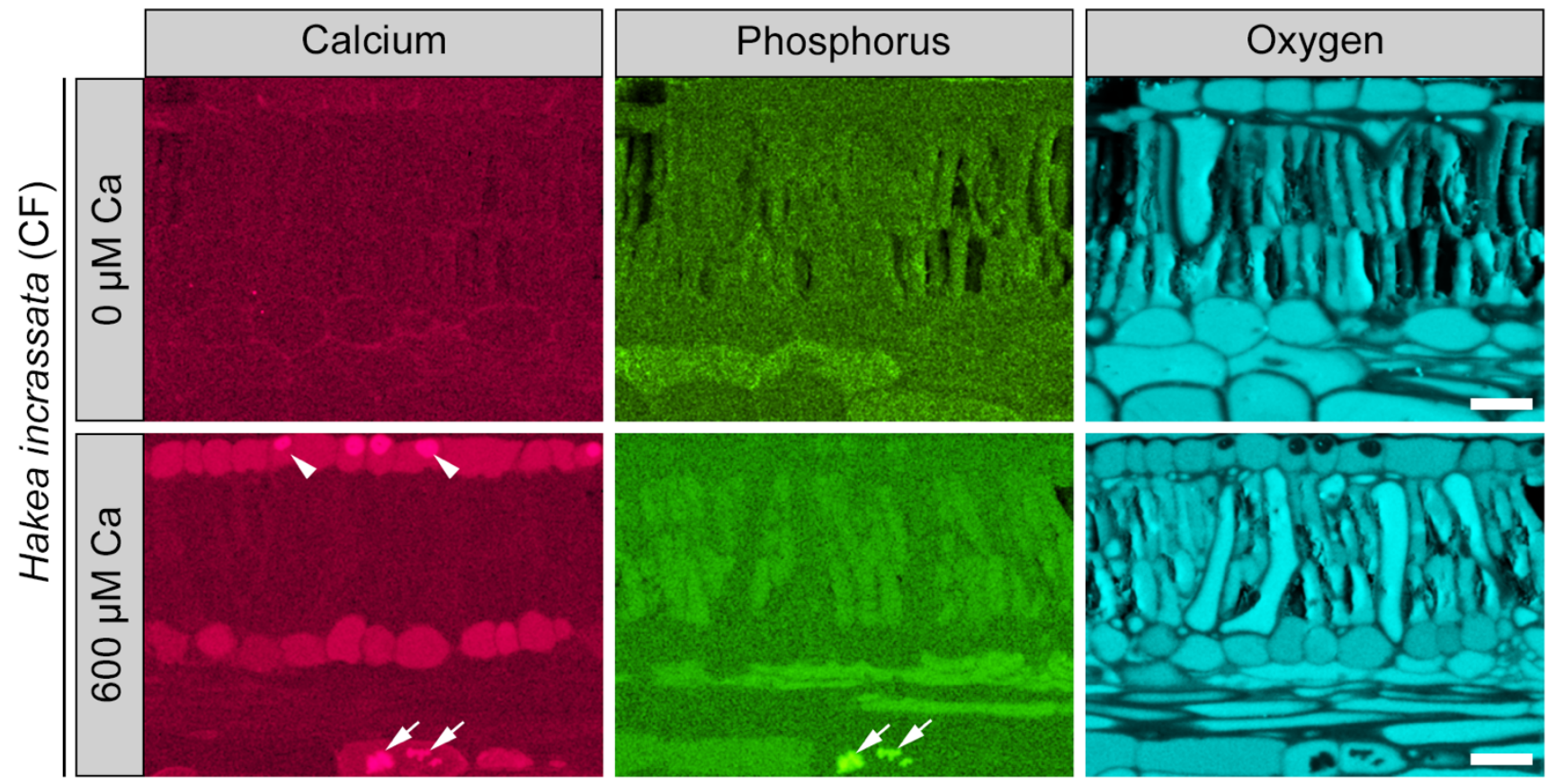

Figure 1. Qualitative element maps showing calcium $(\mathrm{Ca})$, phosphorus $(\mathrm{P})$ and oxygen $(\mathrm{O})$ distributions in transverse leaf sections of the calcifuge (CF) species Hakea incrassata (Proteaceae), grown in hydroponics under two Ca treatments $(0 \mu \mathrm{M}, 600 \mu \mathrm{M})$ and high $\mathrm{P}$ supply $(10 \mu \mathrm{M})$. Phosphorus and Ca maps provide a visualization of the distribution, with quantified concentrations extracted from specific regions of interest within each map. Arrows indicate Ca-phosphate deposits; arrowheads indicate Cabased crystals. Scale bar: $50 \mu \mathrm{m}$. 